\title{
On the advantage of being left-handed in volleyball: further evidence of the specificity of skilled visual perception
}

\author{
Florian Loffing • Jörg Schorer • Norbert Hagemann • \\ Joseph Baker
}

Published online: 7 December 2011

(C) Psychonomic Society, Inc. 2011

\begin{abstract}
High ball speeds and close distances between competitors require athletes in interactive sports to correctly anticipate an opponent's intentions in order to render appropriate reactions. Although it is considered crucial for successful performance, such skill appears impaired when athletes are confronted with a left-handed opponent, possibly because of athletes' reduced perceptual familiarity with rarely encountered left-handed actions. To test this negative perceptual frequency effect hypothesis, we invited 18 skilled and 18 novice volleyball players to predict shot directions of left- and right-handed attacks in a videobased visual anticipation task. In accordance with our predictions, and with recent reports on laterality differences in visual perception, the outcome of left-handed actions was significantly less accurately predicted than the outcome of right-handed attacks. In addition, this left-right bias was most distinct when predictions had to be based on preimpact (i.e., before hand-ball contact) kinematic cues, and skilled players were generally more affected by the opponents'
\end{abstract}

F. Loffing $\cdot$ N. Hagemann

University of Kassel,

Kassel, Germany

J. Schorer

University of Muenster,

Muenster, Germany

J. Baker

York University,

Toronto, Canada

F. Loffing $(\square)$

Institute of Sports and Sports Science, University of Kassel,

Heinrich-Plett-Str. 40,

34132 Kassel, Germany

e-mail: f.loffing@uni-kassel.de handedness than were novices. The study's findings corroborate the assumption that skilled visual perception is attuned to more frequently encountered actions.

Keywords Anticipation · Perceptual expertise . Handedness $\cdot$ Perceptual frequency effect

Left-handed opponents present an interesting obstacle to many athletes in interactive sports. As compared with the general population (10-13\%; Raymond, Pontier, Dufour, \& Møller, 1996), successful left-handers are overrepresented in many interactive sports; for example, in tennis from 1968 to 1999, 34.4\% of males with World Number One rankings were left-handed, despite "lefties" comprising only $\sim 7 \%$ of players (Holtzen, 2000; see also Loffing, Schorer, \& Cobley, 2010). Similar results have been noted in baseball (Goldstein \& Young, 1996; Grondin, Guiard, Ivry, \& Koren, 1999) and cricket (Brooks, Bussière, Jennions, \& Hunt, 2004; for a review, see, e.g., Grouios, Koidou, Tsorbatzoudis, $\&$ Alexandris, 2002). One explanation for this phenomenon is the negative frequency-dependent selection hypothesis proposed by Faurie and Raymond (2005). They supposed that the superiority of left-handed athletes in interactive sports is the result of players having less experience with left-handed opponents. The lack of familiarity with the playing techniques and tactical strategies of left-handed competitors therefore disadvantage a player to act the same as when faced with a right-hander (e.g., Loffing, Hagemann, \& Strauss, 2010). As a result, the inexperience of those players ultimately creates an advantage for their left-handed opponent (cf. Wood \& Aggleton, 1989).

Although a left-handed action may seem to be simply a horizontally inverted version of a right-handed action, assuming that interpreting left- and right-handed actions does not 
involve different cognitive processes may be misleading. Neuropsychological and psychological evidence suggest that the horizontal orientation is an important part of perceiving human motion. Preliminary results in sport studies also support this notion (Hagemann, 2009; McMorris \& Colenso, 1996). On a neuropsychological level, computer tomography has shown that the superior temporal sulcus (STS) is particularly active when individuals perceive human movements (Grossman et al., 2000). Electrophysiological studies using single-cell recording in the STS have revealed that this region also reacts sensitively to the orientation of posture or body movements. For example, Perrett et al. (1985) found cells in the anterior part of the STS (STSa) of a macaque monkey that react to the right-sided profile of a person moving to the right. This same cell does not fire, however, when the left-sided profile of the person moves to the left. Perrett et al. (1985) noted that the majority of cells in the STSa were selective for movement direction and the view of the body, which is specified in relation to the observer (see also Oram \& Perrett, 1996). Cells seem to develop their sensitivity for objects in those conditions in which they usually appear (Perrett, Oram, \& Ashbridge, 1998), indicating that a usual view is an important part in object recognition. Additionally, the tuning to a particular view depends on the amount of experience with the particular viewing circumstance (Perrett et al., 1998). One might speculate on this level that side-specific actions of left- and right-handed people are anisotropic. Therefore, the uneven experience with left- and right-handed postures or movements leads to different adaptations in the regions responsible for human movement recognition.

This specificity in visual movement perception is also reflected in psychological theories. For example, Freyd (1987) proposed that the dynamics of movements are an integral component of their mental representation. Therefore, it can be suggested that side-specific features are also stored in dynamic representations (see Olofsson, Nyberg, \& Nilsson, 1997). Support for this assumption comes from the side-specific priming effects reported by Daems and Verfaillie (1999, Experiments 2 and 3). Using long-term priming studies, Daems and Verfaillie were able to show that the presentation of human body postures during a learning phase (images) lead to faster recognition performance only when the presentations in the test phase take the same horizontal orientation (see also Verfaillie, 1993). Similarly, Stone (1998) tested whether the rotation direction (clockwise or counter clockwise) was an important part of object recognition and found that after a training phase, recognition performance was reduced if rotation direction of novel, three-dimensional objects was reversed. Stone (1998) suggested that the movement direction of objects is stored as an object-specific spatiotemporal signature (also see Stone, 1999).
In interactive sports such as tennis and volleyball, performance depends on sport-specific perceptual or anticipatory skill. In these sports, the ability to exploit the movement information available early in an action sequence (e.g., postural orientation or cues) in order to anticipate an opponent's intention is crucial to successful performance (Farrow \& Abernethy, 2003; Williams \& Ward, 2003). In the past few decades, there has been significant development in our understanding of the perceptual characteristics that differentiate skilled from less-skilled performers (for a detailed review, see Mann, Williams, Ward, \& Janelle, 2007). In general, experts show better performance in perceptionaction coupled situations (Farrow \& Abernethy, 2003), less vulnerability in dual-task requirements (Rowe \& McKenna, 2001), superior recall and recognition of sport-specific patterns of play and of objects (Allard, Graham, \& Paarsalu, 1980), an enhanced ability to effectively pick up advance visual cues (Müller, Abernethy, \& Farrow, 2006), longer quiet-eye durations (Vickers, 2009), and more accurate expectations based on situational probabilities (Abernethy, Gill, Parks, \& Packer, 2001).

One of the first studies to consider the left-hander advantage in sport by analyzing anticipatory skills in an interactive situation was conducted by McMorris and Colenso (1996). They examined perceptual skills of seven English premier league goalkeepers using a temporal occlusion paradigm. Randomized video scenes from two left-footed and two right-footed professional penalty kickers were presented, and goalkeepers had to anticipate the resultant direction of the ball. The results showed that it was easier for goalkeepers to predict ball direction for right-footed players. This finding might be explained by what Neumaier (1983) found earlier in his study of the visual search behavior of German expert and novice volleyball players. Participants responded to video clips showing attacks and lobs of two right-handed and one left-handed player and were instructed to anticipate shot directions. Instead of concentrating on the left arm-shoulder area when the left-handed player was presented, most participants from both groups fixated on the right side, suggesting that task-relevant movement information could not be sufficiently perceived, analyzed, and finally used for anticipation.

Recently, Hagemann (2009) studied whether the greater frequency of tennis matches with right-handed opponents made it possible to discriminate the stroke movements of right-handed players more precisely. Right-handed and lefthanded male tennis players of varying expertise (experts from the first to third German national leagues, intermediates from local leagues, and novices) completed a tennis anticipation test in which they predicted the direction of an opponent's tennis stroke on a computer screen (landing position of the ball in their own half of the court; dependent variable: radial error). They watched video clips of different 
strokes (serves, volleys, and ground strokes) by two rightand two left-handed male intermediate tennis players, with each sequence temporally occluded at racquet-ball contact. Video clips were also presented inverted horizontally, resulting in the same movements seen as a stroke by a right- and a left-handed player. This innovation overcame the methodological problem in previous studies (e.g., McMorris \& Colenso, 1996), in which comparability of test difficulty between left-handers and right-handers was hard to achieve. Hagemann (2009) was able to show that expert, intermediate, and novice tennis players were, irrespective of their own handedness, all better at predicting the direction of strokes by right-handed players. He concluded the overrepresentation of left-handers in expert domain was at least partly due to negative perceptual frequency effects (see also Brooks et al., 2004; Raymond et al., 1996).

An important characteristic of the impact of (negative) perceptual frequency effects has not been verified yet. Namely, given that athletes face more problems anticipating the outcome of left-handed as compared with right-handed actions because of their reduced ability to interpret advanced visual cues provided by left-handed movements, anticipation differences induced by such effects should increase when the amount of advanced visual information is reduced. That is, the left-handers' advantage should be more pronounced when the actions to be anticipated are temporally occluded even before there is contact with a ball (e.g., by the attacking hand in volleyball).

The aim of our present experiment therefore was to better examine the use of advanced visual information as it relates to skilled perception in a volleyball task. To investigate the negative frequency effect, players from the highest volleyball leagues in Germany were compared to volleyball novices. Logically, provided we presented our participants with a representative task (cf. Ericsson \& Smith, 1991), our first assumptions were that skilled volleyball players would outperform novices and that performance would improve with later temporal occlusion conditions (e.g., Farrow, Abernethy, \& Jackson, 2005). Likewise, and on the basis of previous study reports (e.g., Williams \& Burwitz, 1993), we expected that the hypothesized skill effect would be most pronounced when predictions had to be based on early (i.e., pre-hand-ball contact) cues. Moreover, according to the hypothesis of negative perceptual frequency effects (Brooks et al., 2004; Hagemann, 2009; Raymond et al., 1996), we expected that the presentation of left- and right-handed movement sequences would lead to different performances in the anticipatory task, with better scores for right-handed presented actions. To have comparable task difficulties, we used normal and horizontally flipped video clips of left- and right-handed attackers (cf. Hagemann, 2009). We also expected a two-way interaction between expertise of participants and laterality of presented attacks, with stronger negative perceptual frequency effects in skilled players because of (assumed) higher exposure to right-handed attacks. Finally, according to the characteristic of perceptual frequency effects described earlier, we hypothesized that this skill-dependent side bias in information "pick-up" would be affected by the temporal occlusion condition, which should result in a three-way interaction. That is, the left-right difference in prediction performance should be more distinct at early occlusion conditions in skilled players than in novices.

\section{Method}

Participants

Eighteen skilled (age $M=28.11$ years, $S D=8.03$; experience $M=16.33$ years, $S D=8.85 ; 17$ right-handers, 1 lefthander) and 18 novice (age $M=28.56$ years, $S D=8.80 ; 15$ right-handers, 3 left-handers) volleyball players took part in the experiment. Participants' handedness was assessed by a German version of the Edinburgh Handedness Inventory (Oldfield, 1971; laterality quotients: range $_{\mathrm{RH}}=.23-1$; range $_{\mathrm{LH}}=-.80--.30$ ). The skilled players' highest performance was between the first and sixth highest national leagues in Germany, whereas novices had no experience in competitive volleyball. The experiment was conducted according to the Revised Declaration of Helsinki (World Medical Association, 2008).

\section{Apparatus and materials}

As models for the video clips, six male volleyball players (three left- and three right-handers) of medium standard (i.e., playing in the fourth to sixth highest national leagues in Germany) were recruited to perform attacks (also called spikes in volleyball) from three different net positions (i.e., Positions 2, 3 and 4). Each player performed a series of attacks from each position to two directions. Players hit either a down-theline or a diagonal shot from Positions 2 and 4, respectively, whereas from Position 3, they hit the ball left and right toward the sidelines (see Fig. 1). Each attack was introduced by a pass from a teammate in the background at Position 6 to the setter at/near Position 3, who finally set the ball toward the attacking player. Players were filmed from a typical backcourt defender's perspective in the opposing half (i.e., Positions 5, 6, and 1) using a SONY HDR-FX 1000E digital camera (capture rate $25 \mathrm{fps}$ ). For each recording perspective, the camera was placed on a tripod at a height of $1.75 \mathrm{~m}$ and $1.40 \mathrm{~m}$ behind the baseline, so that all players and the complete ball flight were visible throughout an attacking sequence. 
a

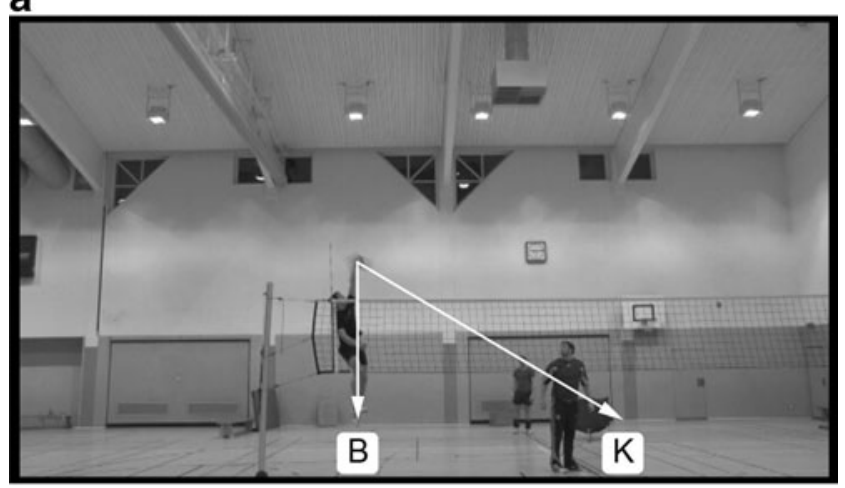

b

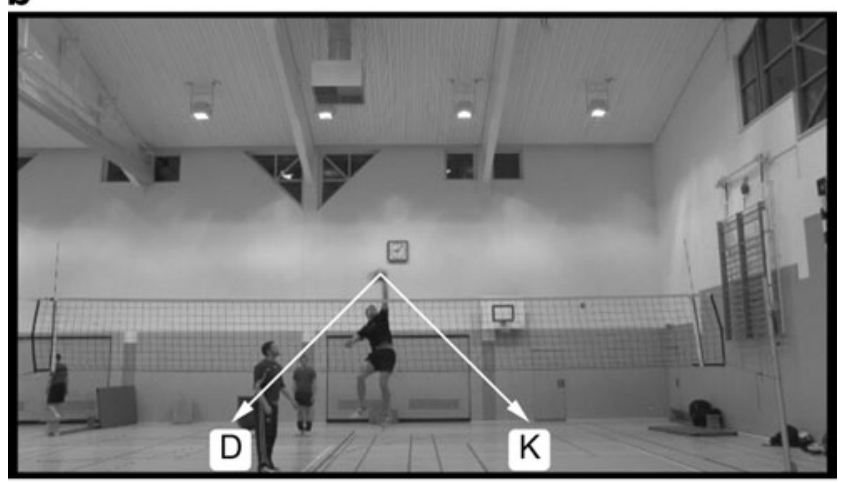

c

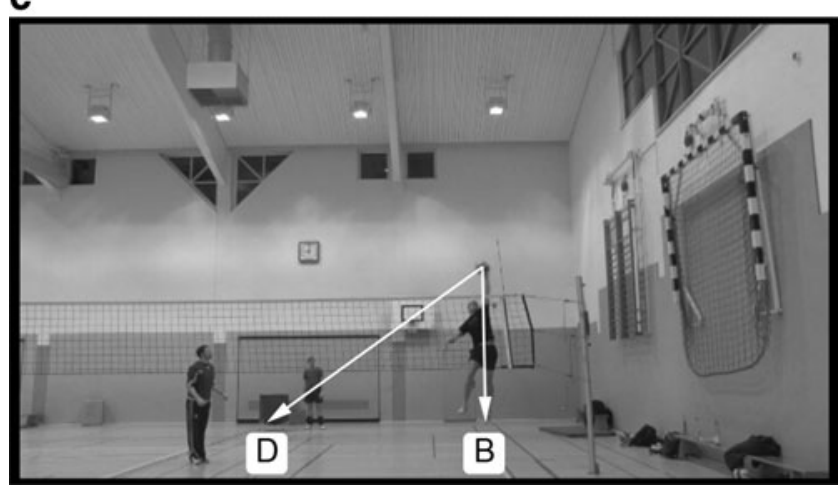

Fig. 1 Illustration of the three different attacking positions presented in the experiment. a Position 2. b Position 3. c Position 4. Pictures show screenshots of the final frame of a video stopping at the moment of hand-ball contact. Arrows indicate shot directions, and characters represent the respective response buttons

Following the recordings, attacks of two left-handed and two right-handed players were selected for the creation of the experimental stimuli. The main criteria for the inclusion of an attack were that shot direction was not predetermined by the attacking player's position on the field, thus limiting the use of event probability information (e.g., only a diagonal shot would have been possible for attacks from Positions 2 and 4 when the attacker was far off the court), and that the moment of an attacker's hand-ball contact could clearly be identified in the footage. From each player, six attacks (i.e., one shot to each direction from each position) were chosen for video editing using Adobe Premiere Pro CS4.
In order to vary the amount of advance visual information and likewise to specify its importance for anticipating shot direction, footage was temporally occluded at three different time points: $\mathrm{T} 1-160 \mathrm{~ms}$ (4 frames) prior to an attacker's hand-ball contact, T2 - $80 \mathrm{~ms}$ ( 2 frames) prior to hand-ball contact, and T3 - moment of hand-ball contact. The duration of clips ending at T3 was $3 \mathrm{~s}$. Furthermore, each video clip was horizontally mirrored so that, for instance, an original right-hander's attack from Position 2 was also displayed as a left-handed attack from Position 4, and vice versa (Hagemann, 2009). This procedure controlled for and eliminated the potential confounding effect of handedness-specific movement patterns (e.g., differences in the ability to perform an attack or disguise one's intentions) on the accuracy of action outcome prediction (cf. McMorris \& Colenso, 1996). Video editing resulted in a total of 144 test clips, with half presenting original attacks and the other half mirrored attacks.

Participants viewed the videos on a 15.4-in. notebook monitor (Gericom Blockbuster Excellent 7000), and the participants' eye-to-screen distance was approximately $50 \mathrm{~cm}$. The videos were shown at a frame rate of $25 \mathrm{fps}$, and video size was 1,280 (width) x 720 (height) pixels.

\section{Task and procedure}

The participants' task was to anticipate the attackers' shot direction by pressing one of two possible response buttons on a QWERTZ keyboard after the end of a video. As is illustrated in Fig. 1, response buttons varied depending on the attackers' shot positions as shot directions also varied depending on attack position (i.e., left vs. right from Position 3; right/left vs. down-the-line from Positions 2/4). In order to prevent participants from screening the final frame of a video, the screen immediately turned black once a sequence had stopped.

Prior to the start of the experiment, participants were given standardized written instructions about the experiment's procedure. They were informed that they would be presented with right- and left-handed actions and that half of these sequences were horizontally mirrored so that the same person was shown attacking both right- and left-handed.

Following the instructions, participants saw 12 familiarization clips showing left- and right-handed attacks of the players not included in the test clips. In order to better illustrate players' position-specific shot direction and task demands, the first six familiarization clips were not temporally occluded. The second half of the clips included attacks occluded as in the experiment. Prior to each familiarization clip, participants were informed about the shot direction of the next attack. After the familiarization phase, participants saw 144 test film sequences. The presentation of original and flipped sequences was blocked and counterbalanced 
across participants of both skill levels, and sequences were randomly presented within blocks. Participants were allowed a short break after completion of half of the experimental clips. After the test procedure, they completed a questionnaire on their experience in sports and their handedness preference for various tasks.

\section{Statistical analyses and dependent variable}

Response accuracy, measured as the percentage of correct predictions, was the dependent variable and was calculated separately for attackers' handedness and temporal occlusion condition. Analysis consisted of a three-way 2 (skill level) $\mathrm{x}$ 2 (attackers' handedness) x 3 (temporal occlusion condition) ANOVA, with repeated measures on the last two factors and with skill level as the between-subjects factor. The Greenhouse-Geisser correction was applied to any violations of sphericity. Alpha was set at .05 , and effect sizes for the ANOVA are reported as partial eta-squared values $\left(\eta_{\mathrm{p}}{ }^{2}\right)$. To address our prediction that the left-right difference in prediction performance should decrease from early to late occlusion conditions, particularly in skilled players, the ANOVA was followed by two-factorial polynomial contrasts on both within-subjects factors (separately for skill level), with focus on the interaction term. As was suggested by Atkinson (2002), we refrained from running multiple comparisons by means of $t$ tests with Bonferroni corrections, since these are likely to increase a Type II error, particularly when small sample sizes are included. Since temporal occlusion condition can be considered an "ordinal" factor (i.e., all levels within this factor can be ordered hierarchically with $\mathrm{T} 1<\mathrm{T} 2<\mathrm{T} 3$ ), use of polynomial contrasts is justified (Atkinson, 2002). Finally, to compare skilled and novice players' performances in each experimental condition with the guessing criterion of $50 \%$, onesample $t$ tests were carried out on mean accuracies for each experimental condition. All analyses were conducted with SPSS 19.

\section{Results}

The ANOVA revealed significant main effects for skill level, $F(1,34)=54.92, p<.001, \eta_{\mathrm{p}}{ }^{2}=.62$, attackers' handedness, $F(1,34)=5.36, p=.03, \eta_{\mathrm{p}}{ }^{2}=.14$, and temporal occlusion condition, $F(1.66,56.47)=99.39, p<.001, \eta_{\mathrm{p}}{ }^{2}=$ .75. Skilled players outperformed novices, participants performed more poorly when confronted with left-handed as compared with right-handed spikes, and performance increased from early to late temporal occlusion conditions. The skill effect was most pronounced at early occlusion conditions, as reflected by a significant skill level $\times$ temporal occlusion interaction, $F(1.66,56.47)=$ $5.50, p=.01, \eta_{\mathrm{p}}{ }^{2}=.14$ (see Fig. 2).

There was no significant skill level $\times$ attackers' handedness interaction, $F(1,34)=.31, p=.58, \eta_{\mathrm{p}}{ }^{2}=.01$. The interaction between attackers' handedness and temporal occlusion approached significance, $F(2,68)=2.74, p=.07$, $\eta_{\mathrm{p}}{ }^{2}=.08$, representing a decrease in performance differences between left- and right-handed attacks with an increasing amount of information available. Moreover, compared with novices, skilled players tended to be more affected by the attackers' handedness across temporal occlusion conditions (see Fig. 3); however, the three-way interaction just failed to reach significance, $F(2,68)=$ $3.10, p=.051, \eta_{\mathrm{p}}{ }^{2}=.08$. Besides significant linear and quadratic trends for temporal occlusion condition in skilled and novices players' performances, polynomial contrasts revealed a significant linear trend for the attackers' handedness by temporal occlusion condition interaction in skilled players only, $F(1,17)=6.54, p=.02, \eta_{\mathrm{p}}^{2}=.20$, whereas in novices, there was a tendency for a quadratic trend for this interaction, $F(1,17)=3.83, p=.07, \eta_{\mathrm{p}}{ }^{2}=.18$.

Overall, skilled and novice participants anticipated shot directions significantly above chance level (i.e., 50\%) in all experimental conditions.

\section{Discussion}

Ericsson and Smith (1991) proposed that a necessary first step in investigating mechanisms of expertise is ensuring the validity of the experimental task. This prerequisite was met, as reflected by the significant main effects of temporal occlusion and skill level. In accordance with results from previous studies (e.g., Farrow et al., 2005), both skilled players and novices performed least accurately at the earliest temporal occlusion condition, and most accurately at the

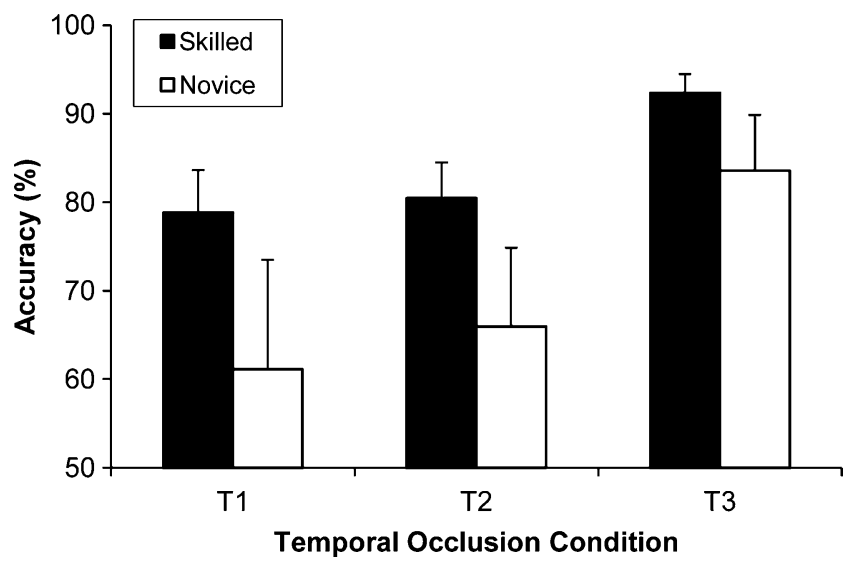

Fig. 2 Mean response accuracies as a function of the participants' skill level and temporal occlusion condition. Error bars indicate standard deviations 

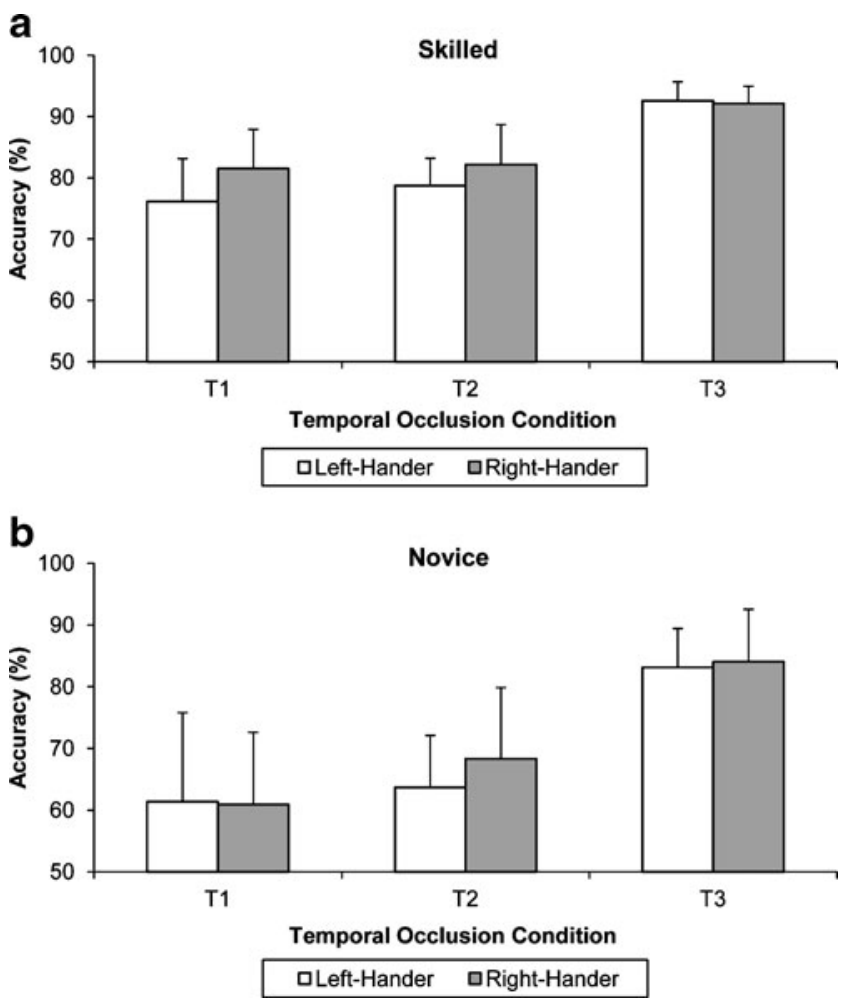

Fig. 3 Mean response accuracies as a function of temporal occlusion condition and the attackers' handedness separately for skilled (a) and novice (b) participants. Error bars indicate standard deviations

latest one. Additionally, and as expected (Mann et al., 2007; Williams, Davids, \& Williams, 1999), skilled players outperformed novices. Furthermore, and again in line with the literature, skilled players could be best distinguished from novices by their performance at early temporal occlusion conditions, as revealed by the significant interaction between the aforementioned factors; however, both groups demonstrated effective (i.e., better than chance) information pick-up in each experimental condition.

These effects allowed us to investigate our hypotheses concerning laterality, confident that we had a valid task. According to the negative perceptual frequency effect hypothesis, and because of reasonable differences in the frequency of left-handers in the normal population and in professional sports, we hypothesized pronounced sidespecific effects. The analysis of response accuracy confirms our assumption and corroborates previous research findings (Hagemann, 2009; McMorris \& Colenso, 1996): Righthanded attacks were significantly better anticipated than left-handed attacks.

There was, however, no significant skill level by attackers' handedness interaction, and the Skill Level $\times$ Attackers' Handedness $\times$ Temporal Occlusion condition just missed the conventional significance level $(p=.051)$. In line with Atkinson (2002, p. 201), and transferred to our data, given the quite small sample size employed presently, "the presence of such an interaction should not be discounted for the population with a $P$-value of" .051 , thus running the risk of making a Type II error. Albeit preliminary, scrutiny of Fig. 3 suggests that the side bias found in skilled players' anticipation of left- and right-handed attacks in volleyball was most evident when predictions had to be based on early kinematic cues. The corresponding significant linear trend for the interaction between attackers' handedness and the temporal occlusion condition found by polynomial contrasts indicates that the left-right difference in response accuracy almost linearly decreased from early to late occlusion conditions (see Fig. 3a). Still, more research is warranted before making a conclusive statement on such a relationship.

A plausible explanation of the significant laterality effect is that observers had greater difficulty interpreting the kinematic interaction of a presented left-handed opponent and, as a result, had lower response accuracies in those situations. Neuropsychological and psychological findings on sidespecific movement representations (cf. Freyd, 1987; Perrett et al., 1985, 1998) provide support for this conclusion. Similarly, perceptual frequency effects would be responsible for a right-hand bias in the situations used in the present experiment and thus are likely to explain (part of) the lefthander advantage in interactive sports (cf. Brooks et al., 2004; Faurie \& Raymond, 2005; Hagemann, 2009). However, although there is growing evidence to suggest that the benefit of performing left-handed in sports is mainly the result of a reduced familiarity with the left-handers' movements and game play behavior (Loffing et al., 2010), other factors such as beneficial genetic or neuropsychological predispositions in left-handers still need to be ruled out (for such an explanation, see, e.g., Holtzen, 2000).

Although the present results are, for the most part, in accordance with the expectations following from the negative perceptual frequency effects hypothesis, there is a limitation regarding the generalization of findings across left- and right-handed observers, since only four left-handed individuals were included in this study. Considering the ongoing debate on which factors (i.e., visual vs. motor experience) primarily contribute to participants' performance in visual perceptual tasks (e.g. Aglioti, Cesari, Romani, \& Urgesi, 2008; Jacobs, Pinto, \& Shiffrar, 2004), one might expect that the observer's handedness moderates anticipation of lateralized actions and that congruence between an observer's manual preference and a model's performing side facilitates performance (Gardner \& Potts, 2010). Clearly, we cannot rule out that the observer's handedness did not have an impact on prediction performance in our experimental task. However, the findings by Hagemann (2009) give reason to assume that anticipation of the path of an object that will be, or is, directed through a lateralized action is independent of the observers' manual dominance. 
Therefore, we refrained from including left-handed participants as a group into the protocol. Nonetheless, since recent evidence also suggests that observers rely on different strategies, depending on the perceptual task (e.g., recognition of human movements vs. anticipating the effects of human movements; Cañal-Bruland \& Williams, 2010), it appears worthwhile that future research determine the contribution of our (lateralized) motor system on various perceptual tasks.

To conclude, findings indicating side-specific visual perception support the assumption that the orientation of action presentation has a considerable influence on athletes' anticipation performance in interactive sporting situations. Even at the early developmental stages of perceptual expertise, highly specific adaptations occur to the cognitive system, and after years of practice, these adaptations become highly specific (Ericsson \& Lehmann, 1996). Further research, however, is needed to reconcile these findings with previous work suggesting that experts are better able to transfer pattern recall or recognition skills from their domain to structurally related domains than are less skilled performers (e.g., Abernethy, Baker, \& Côté, 2005; Smeeton, Ward, \& Williams, 2004). It is possible that transfer of expertise is limited to a specific subset of cognitive skills. Collectively, the present results provide increased evidence for the specificity of skilled visual perception in sport.

Author note We thank Sarah Jakob for her helping hand during data collection.

\section{References}

Abernethy, B., Baker, J., \& Côté, J. (2005). Transfer of pattern recall skills may contribute to the development of sport expertise. Applied Cognitive Psychology, 19, 705-718.

Abernethy, B., Gill, D. P., Parks, S. L., \& Packer, S. T. (2001). Expertise and the perception of kinematic and situational probability information. Perception, 30, 233-252.

Aglioti, S. M., Cesari, P., Romani, M., \& Urgesi, C. (2008). Action anticipation and motor resonance in elite basketball players. Nature Neuroscience, 11, 1109-1116.

Allard, F., Graham, S., \& Paarsulu, M. E. (1980). Perception in sport: Basketball. Journal of Sport Psychology, 2, 14-21.

Atkinson, G. (2002). Analysis of repeated measurements in physical therapy research: Multiple comparisons amongst level means and multi-factorial designs. Physical Therapy in Sport, 3, 191-203.

Brooks, R., Bussière, L. F., Jennions, M. D., \& Hunt, J. (2004). Sinister strategies succeed at the cricket World Cup. Proceedings of the Royal Society of London B, 271, 64-66.

Cañal-Bruland, R., \& Williams, A. M. (2010). Recognizing and predicting movement effects: Identifying critical movement features. Experimental Psychology, 57, 320-326.

Daems, A., \& Verfaillie, K. (1999). Viewpoint-dependent priming effects in the perception of human actions and body postures. Visual Cognition, 6, 665-693.
Ericsson, K. A., \& Lehmann, A. C. (1996). Expert and exceptional performance: Evidence of maximal adaptation to task constraints. Annual Review of Psychology, 47, 273-305.

Ericsson, K. A., \& Smith, J. (1991). Prospects and limits of the empirical study of expertise: An introduction. In K. A. Ericsson \& J. Smith (Eds.), Toward a general theory of expertise: Prospects and limits (pp. 1-38). Cambridge, MA: Cambridge University Press.

Farrow, D., \& Abernethy, B. (2003). Do expertise and the degree of perception-action coupling affect natural anticipatory performance? Perception, 32, 1127-1139.

Farrow, D., Abernethy, B., \& Jackson, R. C. (2005). Probing expert anticipation with the temporal occlusion paradigm: Experimental investigations of some methodological issues. Motor Control, 9, $332-351$.

Faurie, C., \& Raymond, M. (2005). Handedness, homicide and negative frequency-dependent selection. Proceedings of the Royal Society of London B, 272, 25-28.

Freyd, J. J. (1987). Dynamic mental representations. Psychological Review, 94, 427-438.

Gardner, M. R., \& Potts, R. (2010). Hand dominance influences the processing of observed bodies. Brain and Cognition, 73, 35-40.

Goldstein, S. R., \& Young, C. A. (1996). "Evolutionary" stable strategy of handedness in major league baseball. Journal of Comparative Psychology, 110, 164-169.

Grondin, S., Guiard, Y., Ivry, R. B., \& Koren, S. (1999). Manual laterality and hitting performance in major league baseball. Journal of Experimental Psychology: Human Perception \& Performance, $25,747-754$.

Grossman, E., Donnelly, M., Price, R., Pickens, D., Morgan, V., Neighbor, G., et al. (2000). Brain areas involved in perception of biological motion. Journal of Cognitive Neuroscience, 12, $711-720$

Grouios, G., Koidou, I., Tsorbatzoudis, H., \& Alexandris, K. (2002). Handedness in sport. Journal of Human Movement Studies, 43, 347-361.

Hagemann, N. (2009). The advantage of being left-handed in interactive sports. Attention, Perception, \& Psychophysics, 71, $1641-1648$.

Holtzen, D. W. (2000). Handedness and professional tennis. International Journal of Neuroscience, 105, 101-119.

Jacobs, A., Pinto, J., \& Shiffrar, M. (2004). Experience, context, and the visual perception of human movement. Journal of Experimental Psychology: Human Perception \& Performance, 30, 822-835.

Loffing, F., Hagemann, N., \& Strauss, B. (2010). Automated processes in tennis: Do left-handed players benefit from the tactical preferences of their opponents? Journal of Sports Sciences, $28,435-443$.

Loffing, F., Schorer, J., \& Cobley, S. P. (2010). Relative age effects are a developmental problem in tennis: But not necessarily when you're left-handed! High Ability Studies, 21, 19-25.

Mann, D. T. Y., Williams, A. M., Ward, P., \& Janelle, C. M. (2007). Perceptual-cognitive expertise in sport: A meta-analysis. Journal of Sport \& Exercise Psychology, 29, 457-478.

McMorris, T., \& Colenso, S. (1996). Anticipation of professional soccer goalkeepers when facing right- and left-footed penalty kicks. Perceptual and Motor Skills, 82, 931-934.

Müller, S., Abernethy, B., \& Farrow, D. (2006). How do world-class cricket batsmen anticipate a bowler's intention? Quarterly Journal of Experimental Psychology, 59, 2162-2186.

Neumaier, A. (1983). Beobachtungsstrategien und Antizipation bei der Abwehr von Volleyballangriffen. [Observational strategies and anticipation during the defence of attacks in volleyball.]. Leistungssport, 13, 5-10.

Oldfield, R. C. (1971). The assessment and analysis of handedness: The Edinburgh inventory. Neuropsychologia, 9, 97-113. 
Olofsson, U., Nyberg, L., \& Nilsson, L.-G. (1997). Priming and recognition of human motion patterns. Visual Cognition, 4, 373382.

Oram, M. W., \& Perrett, D. I. (1996). Integration of form and motion in the anterior superior temporal polysensory area (STPa) of the macaque monkey. Journal of Neurophysiology, 76, 109-129.

Perrett, D. I., Oram, M. W., \& Ashbridge, E. (1998). Evidence accumulation in cell populations responsive to faces: An account of generalisation of recognition without mental transformations. Cognition, 67, 111-145.

Perrett, D. I., Smith, P. A., Mistlin, A. J., Chitty, A. J., Head, A. S., Potter, D. D., et al. (1985). Visual analysis of body movements by neurones in the temporal cortex of the macaque monkey: A preliminary report. Behavioural Brain Research, 16, 153-170.

Raymond, M., Pontier, D., Dufour, A. B., \& Møller, A. P. (1996). Frequency-dependent maintenance of left handedness in humans. Proceedings of the Royal Society of London B, 263, 1627-1633.

Rowe, R. M., \& McKenna, F. P. (2001). Skilled anticipation in realworld tasks: Measurement of attentional demands in the domain of tennis. Journal of Experimental Psychology: Applied, 7, 6067.

Smeeton, N. J., Ward, P., \& Williams, A. M. (2004). Do pattern recognition skills transfer across sport? A preliminary analysis. Journal of Sports Sciences, 22, 205-213.

Stone, J. V. (1998). Object recognition using spatiotemporal signatures. Vision Research, 38, 947-951.
Stone, J. V. (1999). Object recognition: View-specificity and motionspecificity. Vision Research, 39, 4032-4044.

Verfaillie, K. (1993). Orientation-dependent priming effects in the perception of biological motion. Journal of Experimental Psychology: Human Perception and Performance, 19, 992-1013.

Vickers, J. N. (2009). Advances in coupling perception and action: The quiet eye as a bidirectional link between gaze, attention, and action. In M. Raab, J. G. Johnson, \& H. R. Heekeren (Eds.), Progress in Brain Research (Vol. 174, pp. 279-288).

Williams, A. M., \& Burwitz, L. (1993). Advance cue utilisation in soccer. In T. Reilly, J. Clarys, \& A. Stibbe (Eds.), Science and football II (pp. 239-244). London, England: E. \& F.N. Spon.

Williams, A. M., Davids, K., \& Williams, J. G. (1999). Visual perception and action in sport. London, England: E. \& F.N. Spon.

Williams, A. M., \& Ward, P. (2003). Perceptual expertise: Development in sport. In J. L. Starkes \& K. A. Ericsson (Eds.), Expert performance in sports: Advances in research on sport expertise (pp. 219-249). Champaign, IL: Human Kinetics.

Wood, C. J., \& Aggleton, J. P. (1989). Handedness in "fast ball" sports: Do left-handers have an innate advantage? British Journal of Psychology, 80, 227-240.

World Medical Association. (2008). Declaration of Helsinki-Ethical principles for medical research involving human subjects. Retrieved May 10, 2009, from http://www.wma.net/en/30publications/ 10policies/b3/index.html 